\title{
Técnicas e instrumentos de recolección de información: análisis y procesamiento realizado por el investigador cualitativo
}

Fecha de recepción : 2020-11-18 • Fecha de aceptación: 2020-12-16 • Fecha de publicación: 2021-10-01

Maream J. Sánchez ${ }^{1}$

Universidad Nacional Experimental Rafael María Baralt, Venezuela mareamjsb1@hotmail.com

https://orcid.org/0000-0002-4518-9631

Mariela Fernández ${ }^{2}$

Universidad Nacional Experimental Rafael María Baralt, Venezuela marifer3036@hotmail.com

https://orcid.org/0000-0002-0299-8578

Juan C. Diaz

Universidad Politécnica Territorial del Zulia, Venezuela juancdg80@gmail.com

https://orcid.org/0000-0002-1984-7768

\section{Resumen}

El propósito de este articulo radica en analizar e interpretar la pluralidad de las perspectivas que nos muestran los puntos de vista coincidentes y contrapuestos entre el investigador y los sujetos, siendo paradójicamente uno de los aspectos que enriquece y nutre de complejidad y diversidad de técnicas e instrumentos de recolección de información en el análisis y procesamiento de la investigación cualitativa. Entre las técnicas de recolección de información consideradas se destacan la observación participante, el grupo nominal, Delphi, entrevista a profundidad, grupos focales, revisión documental, taller investigativo y técnicas proyectivas, y en cuanto los instrumentos se recalcan la guía de observación, anecdotarios, diario del investigador y artefactos. En relación al análisis y procesamiento de 
la información indagada se enfatizan la inducción analítica, método comparativo constante, codificación, descripción densa, análisis de contenido y análisis del discurso, perspectiva de mapeo y la triangulación. Todas y cada una de ellas permiten revelar como piensan, sienten y actúan los sujetos involucrados en una investigación, razón principal por la que es primordial aplicar cada técnica e instrumento adecuadamente, proporcionando una enorme riqueza informativa que pueden corregir los sesgos propios de cada método.

\title{
Palabras clave: técnicas e instrumentos, análisis y procesamiento, cualitativo, investigador.
}

\begin{abstract}
The purpose of this article is to analyze and interpret the plurality of perspectives that show us the coincident and opposed points of view between the researcher and the subjects, being paradoxically one of the aspects that enriches and feeds the complexity and diversity of techniques and instruments of information collection in the analysis and processing of qualitative research. Among the techniques of information collection considered, the following stand out: participant observation, nominal group, Delphi, in-depth interview, focal groups, documentary review, research workshop and projective techniques, and as far as the instruments are concerned, the observation guide, anecdotes, researcher's diary and artifacts. In relation to the analysis and processing of the investigated information, the analytical induction, constant comparative method, codification, dense description, content analysis and discourse analysis, mapping perspective and triangulation are emphasized. Each and every one of them allows revealing how the subjects involved in an investigation think, feel and act, which is the main reason why it is essential to apply each technique and instrument adequately, providing an enormous wealth of information that can correct the biases inherent to each method.
\end{abstract}

Keywords: techniques and instruments, analysis and processing, qualitative, researcher. 


\section{Introducción}

La investigación cualitativa tiene un potencial muy importante para el estudio de la sociedad. Es un tipo de metodología que ha ganado espacio en los círculos académicos de nuestra Universidad Nacional Experimental Rafael María Baralt (UNERMB), de Venezuela. Razón principal por la que entendemos que una de las tareas cruciales es el manejo de las técnicas la recolección de información, al igual que el análisis de la misma. Evidentemente, las técnicas e instrumentos en la investigación cualitativa proporcionan una mayor profundidad de búsqueda; ante una situación social que amerita ser conocida.

Es necesario, como profesores universitarios investigadores, encontrar una armonía entre el contexto de las técnicas e instrumentos de recolección de información, análisis y procesamiento de la misma mediante una visión clara de la investigación. Es decir, que el conocimiento científico este en equilibrio con las técnicas que son imperativas para cada investigación, según el método o tradición en la cual se desarrolle, siendo el punto trascendental para cualquier proceso investigativo.

Precisamente, tal punto de vista, conlleva el analizar e interpretar aquella pluralidad de perspectivas, los cuales nos muestran los puntos de vista, a veces coincidentes, a veces contrapuestos entre el investigador y los sujetos. Paradójicamente, lo anterior es uno de los aspectos de la investigación cualitativa que la enriquece, la nutre de complejidad y diversidad del proceso investigativo.

Es de considerar que el investigador cualitativo se plantea ciertos enigmas: ¿cómo ingresó al campo y recolectó la información?; ¿cómo almacenó lo recabado?; ¿cómo analizo? y, ¿cómo los procesó? Partiendo de estas interrogantes el ensayo se enmarca en la idea general de conocer ese abanico científico de las técnicas e instrumentos para la recolección de la información y análisis de las mismas, ya que la UNERMB, mediante los diferentes seminarios en especializaciones, maestrías y doctorados, dan a conocer para que se consoliden estudios minuciosos de calidad.

Es importante señalar que, las técnicas más usadas, se centran en el lenguaje hablado o escrito; pero el lenguaje sirve tanto para revelar lo que pensamos y sentimos, como también para ocultarlo, por tal razón debemos ser analíticos y reflexivos desde la aplicación de cada técnica un instrumento adecuado, proporcionando una enorme riqueza informativa que pueden corregir los sesgos propios de cada método.

\section{Metodología}

Las técnicas cualitativas aluden a procedimientos de actuación concreta y particular de recogida de información relacionada con el método de investigación que se está utilizando. De hecho, según refiere Campoy y Gomes (2009: 275), la selección de las técnicas es independiente del enfoque epistemológico del investigador. Si bien es cierto que las técnicas cualitativas han recibido numerosas críticas basadas en la presunta falta de objetividad, la imposibilidad de reproducción de sus datos y la falta de validez. 
De hecho, esas críticas provocaron en los investigadores cualitativos una posición de inferioridad y de falta de reconocimiento; sin embargo, en la actualidad la metodología cualitativa se ha fortalecido como procedimiento que pretende la obtención de conocimiento científico a través de una gran variabilidad de técnicas, entre las cuales se destacan:

\section{La observación participante}

Todos los fenómenos y sucesos que ocurren en un lugar o escenario son objeto de observación. Precisamente, "observar es un proceso que requiere atención voluntaria, selectiva, inteligente, orientado por un proceso terminal u organizador" (Santos, G., 1993:12). En este sentido, se puede considerar que esta técnica es la piedra angular de los métodos de investigación cualitativa, ya que observar no consiste simplemente en mirar, sino en buscar.

El asumir esa condición exige un principio estructurador de la mirada y del pensamiento; entonces, es imperante desde los seminarios impartidos en la universidad y los cuales aluden a los procesos investigativos, la necesidad de promover el educar los ojos para ver las realidades y las raíces epistémicas de un tema de investigación que se genera en un contexto.

En este punto se puede distinguir la observación participante, que según Santana L. (2010: 290) es una técnica de recogida de información en la cual el observador participa en la vida del grupo u organización que estudia, es decir, el investigador se sumerge en el escenario y en la vida de los sujetos, objeto de estudio para oír, ver y desarrollar como los sujetos que la viven, que implica la intervención directa del observador de forma que el investigador puede intervenir en la vida del grupo.

\subsection{Grupo nominal}

Para hablar sobre esta técnica, es necesario acotar que la misma se convierte por méritos propios en una técnica de investigación cualitativa presente en diferentes campos de las ciencias sociales, porque "se manifiesta la cooperación orientada al diagnóstico, tratamiento e intervención de ciertas problemáticas, además de la generación de un compromiso en el espacio apostando por la homogeneidad del grupo" (Hernández, M. 2009:43).

Desde tal planteamiento, se puede decir que dicha técnica facilita la generación de ideas y el análisis de problemas, resulta útil para las situaciones en que las opiniones individuales deber ser combinadas para llegar a decisiones las cuales no pueden o no conviene que sean tomadas por una sola persona, permitiendo la identificación y jerarquizaron de problemas, causas o soluciones a través de consenso en grupos o equipos de trabajo.

\subsection{Técnica Delphi}

La técnica Delphi fue desarrollada en 1950 por Dalkey y colaborados y tiene por objetivo conocer la opinión de un grupo de personas en relación a un problema, sin que los integrantes se reúnan 
físicamente. Linston y Turoff definen la técnica Delphi como un método de estructuración de un proceso de comunicación grupal que es efectivo a la hora de permitir a un grupo de individuos, como un todo, tratar un problema complejo (Campoy A. y Gomes E., 2009: 284).

En dicha técnica convergen una serie de personas considerados expertos, con el fin de obtener un consenso frente a una temática o problemática en común, generalmente se consulta a personas con experiencia, diferente formación y/o jerarquía. Esta técnica es interactiva, porque se puede establecer una comunicación grupal remota o a distancia en que los participantes no tienen comunicación directa cara a cara, y estructurada porque se establecen una serie de cuestionarios aplicados en forma secuencial en el tiempo.

Cabe resaltar que esta técnica, a pesar de no ser nueva, es una de las menos utilizadas, y es necesario en estos momentos de aislamiento social, donde la educación es remota, se considera como una alternativa articulada con el desarrollo actual de las telecomunicaciones, los cuestionarios se pueden administrar por e-mail, fax, web, plataformas online, entre otras.

\subsection{Entrevista a profundidad}

Antes de explicar la técnica de la entrevista en profundidad, se debe abordar la temática de la entrevista en el ámbito de la investigación cualitativa, considerando por supuesto, el hecho incuestionable que el mismo ocurre a través del uso del lenguaje, cuya universalidad es innegable en cualquier esfera de ámbito social. En un sentido general, Campoy A. y Gomes E. (2009: 288) explican que esta técnica se entiende como una interacción entre dos personas, planificada y que obedece a un objetivo, en la que el entrevistado da su opinión sobre un asunto y, el entrevistador, recoge e interpreta esa visión particular.

De hecho, en la metodología cualitativa, tal como lo mencionan los dichos autores, la entrevista en profundidad se entiende como los encuentros reiterados cara a cara entre el investigador y el entrevistado, que tienen como finalidad conocer la opinión y la perspectiva que un sujeto tiene respecto de su vida, experiencias o situaciones vividas. Es de resaltar que esta técnica adopta diferentes clasificaciones como: estructuradas; semiestructuradas y no estructuradas.

\subsection{Grupo Focal}

Se utiliza de forma grupal, es decir, el grupo focal "es un método de investigación colectivista, más que individualista, y se centra en la pluralidad y variedad de las actitudes, experiencias y creencias de los participantes, y lo hace en un espacio de tiempo relativamente corto" (Martínez, M 1999: $56)$.

En concordancia con el autor, se infiere que el grupo focal busca generar un espacio y situación de interacción cómodo y natural para las personas, donde exista una intermediación de la persona moderadora, ya que el éxito de esta técnica depende de que el desarrollo de la entrevista grupal pueda propiciar una conversación que arroje información con el detalle y profundidad. 


\subsection{Revisión documental}

La revisión documental resulta de mucho interés; ya que esta "constituye el punto de entrada a la investigación, incluso en ocasiones es el origen del tema o problema de investigación. Los documentos fuente pueden ser de naturaleza diversa: personales, institucionales o grupales, formales o informales" (Quintana 2006:34).

Por medio de esta es posible obtener información valiosa para lograr el encuadre que incluye, básicamente, describir los acontecimientos rutinarios, así como los problemas y reacciones más usuales de las personas o cultura objeto de análisis, así mismo, conocer los nombres e identificar los roles de las personas es clave en esta situación sociocultural. Revelar los intereses y las perspectivas de comprensión de la realidad, que caracterizan a los que han escrito los documentos.

\subsection{Taller investigativo}

Es una de las menos utilizadas, es de destacar que esta comparte muchos de los aspectos parecidos a los grupos focales, en cuanto a las características de los actores que son convocados. Incluso, en cierta forma, se podría decir que un taller es un espacio de trabajo compartido por dos o hasta tres grupos focales simultáneamente.

La técnica anteriormente mencionada en particular reviste gran importancia en los proyectos de investigación-acción participativa. Brinda la posibilidad de abordar, desde una perspectiva integral y participativa, problemáticas sociales que requieren algún cambio o desarrollo.

Sustentando esta técnica se hace mención a González (1987), quien conceptualiza al taller investigativo como "un espacio-tiempo para la vivencia, la reflexión y la conceptualización y búsqueda de información como síntesis del pensar, el sentir y el hacer desde el describir o caracterizar el problema de estudio dentro de un contexto particular."

\subsection{Técnicas proyectivas}

Existe una gran gama de posibilidades en cuanto a estas técnicas, pero primeramente se debe tener en cuenta que "estas técnicas pueden usarse para incitar de forma indirecta a los participantes a proyectar sus motivaciones, creencias, actitudes o sentimientos subyacentes con respecto a los temas de interés” (Ibáñez, 1992). Entre esa gama se puntualizan las siguientes:

- Asociación: técnica en la cual se presenta un estímulo al participante y se le pide que responda lo primero que le venga a la mente.

- Construcción: técnica en la que los participantes deben construir una respuesta en forma de historia, diálogo o descripción. 
- Expresión: técnica en la que se presenta al participante una situación verbal o visual, y se le pide que relacione los sentimientos y las actitudes de otras personas con la situación.

- Complementación: se pide a los participantes que complementen una situación de estímulo incompleta.

\subsection{Profundizando en el tema de los instrumentos}

En esa búsqueda de técnicas para la recolección de información, el seminario investigación cualitativa ha permitido ampliar los conocimientos en cuanto a las técnicas proyectivas, las cuales permiten una búsqueda de información al interpretar la conducta de otros, los participantes reflejan de manera indirecta sus propias motivaciones, creencias, actitudes o sentimientos en la situación.

En este punto, es necesario resaltar lo propuesto por Arias (2016: 98) quien plantea que los instrumentos "son las distintas formas o maneras de obtener la información". Al considerarlo en la investigación cualitativa, son aquellos medios que se utilizan para recopilar la información que proviene de la aplicación de una técnica determinada con el propósito de registrar las relaciones sociales y describir la realidad como lo experimentan sus correspondientes protagonistas.

Algunos de los instrumentos que se consideraron más relevantes se resaltan:

\subsection{Formatos de observación}

Se debe recordar que el observador tiene un papel muy activo en la indagación cualitativa y su rol puede adquirir diferentes niveles de participación, dependiendo el tipo de investigación. Asimismo, se debe tener en cuenta que los formatos de observación son conocidos también como guías y formularios.

Para Cuevas (2009) en (Hernández y otros 2010:414) señala que como investigadores sabemos que debemos observar y anotar todo lo que consideremos pertinente, y el formato puede ser tan simple como una hoja dividida en dos, un lado donde se registran las anotaciones descriptivas de la observación y otra las interpretativas. Conforme avanza la inducción el proceso investigativo, se pueden ir generando listados de elementos que no podemos dejar fuera, y unidades que deben analizarse.

Por un lado, después de la inmersión inicial, y de que sabemos en qué elementos enfocarnos, se pueden diseñar ciertos formatos de observación, que pueden incluir, por ejemplo; nombre del estudio, propósito, episodio o situación, fecha, hora, participantes, tema principal, impresiones e inclusive un resumen de lo sucedido. Por otro lado, se debe preparar una lista con afirmaciones o preguntas, que orientan el trabajo de observación señalando los aspectos que son relevantes al observar. 


\subsection{Registros anecdóticos o anecdotarios}

Con este tipo de instrumento, como investigadores podemos recoger las mayores impresiones de los sujetos inmersos en la misma investigación. De acuerdo a Morocho (2011), citado por Ramírez $\mathrm{K}(2017: 8)$, el registro anecdótico es un informe que describe hechos, sucesos o situaciones concretas que se consideran importantes para un grupo, y da cuenta de sus comportamientos, actitudes, intereses o procedimientos. De allí que resulta importante poder registrar y sacar provecho a los hechos más significativos y conservar algunas ideas.

Cada situación debe registrarse y describirse de forma breve, para tal fin, se requiere utilizar una libreta, un cuaderno o unas fichas donde se escriba la fecha, nombre, genero, edad, momento, anécdotas, descripción de lo observado y análisis. Esto permite reconocer y valorar diferentes perspectivas, opiniones y posturas frente a un hecho o una situación.

\subsection{Diario de campo}

También conocido como diario del investigador, es un instrumento ideal para el registro de información en las investigaciones cualitativas. Para Hernández y otros (2006), resulta ser un instrumento muy utilizado por los investigadores para registrar aquellos hechos que son susceptibles de ser interpretados. En ese sentido, se puede considerar una herramienta que permite sistematizar las experiencias para luego analizar los resultados.

De hecho, se puede utilizar desde el mismo inicio de un estudio, tomando notas de manera organizativa: fechas y lugar de encuentros, nombre de actividades e involucrados, descripción, reflexiones, entre otros aspectos que se consideren relevantes.

\subsection{Registro de hechos}

Los registros de hechos están relacionados a cualquier objeto fabricado para desempeñar alguna función específica, como captar una imagen, la voz, los sonidos; y pueden servir de evidencia en una investigación cualitativa. Entre estos se pueden mencionar las cámaras fotográficas, teléfonos móviles, dispositivos tecnológicos, filmadoras, entre otros. Sin embargo, en esta oportunidad se puntualizarán algunos, según lo resalta Vargas (2010:46):

- La fotografía: es una técnica muy usada en diversos tipos de investigación, sobre todo la cualitativa. Tiene la característica de captar la realidad de manera fija.

- El video: permite grabar secuencias de imágenes de la realidad, pudiendo así reproducir el movimiento de cosas y personas para su estudio detallado después e inclusive permite volver a ella cuantas veces sea necesario a fin de revisar una y otra vez lo sucedido en una escena en particular. 
- El audio-grabación: es una técnica muy útil cuando se hacen entrevistas, aunque se tomen algunas notas, porque siempre es posible reproducir fragmentos y escuchar de nueva cuenta lo grabado.

También es de gran riqueza poder escuchar el audio de una entrevista varias veces porque cada vez que se escucha se logran significados que en la primera vez no se había captado. Todos y cada uno de estos instrumentos resultan de gran utilidad y respaldo de la información recabada durante el proceso investigativo, como evidencia del trabajo realizado.

\section{Resultados}

Para lograr la investigación fue necesario utilizar diversas técnicas o herramientas de calidad total que permitieron canalizar toda la información. En consecuencia, con la etapa reflexiva mediante el análisis de la realidad que permito dar respuesta a los propósitos que se estimaron necesarios para lograr la cosmovisión consensuada en de los sujetos participantes en la investigación desde procesos dialógicos enmarcados en la discusión-reflexión sobre el trabajo realizado logrando una reconstrucción del mismo, que permitió una visión global profunda del proceso.

Fase de planificar: se orienta hacia la acción. Se debe decidir, pensar en la preocupación temática y en las posibilidades, limitaciones de tal situación, para conocer qué se puede hacer para mejorar. De acuerdo a ello, la fase de planificación permitió, la selección de la problemática.

Fase de actuar: se planea la acción a emprender, debe examinarse la situación en términos de condiciones objetivas (oportunidades, limitaciones físicas y materiales en el marco de su situación, disponibilidad de recursos, límites, tiempo, espacio) condiciones subjetivas (oportunidades restricciones en cuanto al modo en que la gente piensa actualmente, a las expectativas, pautas existentes de relaciones formales e informales, entre otras.

Fase de reflexionar: se sugiere que piense sintéticamente, que intente integrar sus ideas en las categorías del lenguaje/discurso, acción/práctica y relaciones sociales/organización.

\section{Profundizando en el análisis y la interpretación de la información}

En este punto se aclara que no hay recetas para que el investigador lleve a cabo diferentes formas de investigación cualitativa, este es lo más cercano a un artista, y ese arte científico es promovido en la universidad caso de estudio, como una premisa. Sin embargo, lo anteriormente mencionado no puede servir para justificar el caos en una investigación, esas formas no pueden ser anárquicas, del tipo vale todo, porque terminan siendo confusas, lo que va en detrimento de la validez y credibilidad del mismo estudio.

Razón suficiente para encarar la tarea de analizar e interpretar la información significa asumir posturas, tanto académicas, como ideológicas y nos enfrenta a constantes desafíos y dificultades propias de su naturaleza. 
La gran cantidad de información, lo irrepetible de los fenómenos, la cercanía con el sentido común, la necesidad de lograr riqueza analítica y profundidad narrativa, hacen que el análisis sea una tarea que se presenta como un reto apasionante.

Precisamente, en la investigación cualitativa la recolección y el análisis ocurren prácticamente en paralelo; además, el análisis no es estándar, ni una receta, ya que cada estudio requiere de un esquema propio y se pone en manifiesto la creatividad del investigador.

En ese sentido, se hace alusión solo a algunas técnicas de análisis:

\subsection{Bitácora de análisis}

Para el investigador cualitativo, la reflexión es indispensable, por esto es tan importante esta herramienta. Según Hernández y otros (2006: 447) la bitácora tiene la función de documentar el procedimiento de análisis y las propias reacciones del investigador a través de un proceso y contiene fundamentalmente las notaciones sobre el método utilizado, donde se describe el proceso y cada actividad realizada, las ideas, conceptos, significados y categorías que van surgiendo, la credibilidad y verificación del estudio, para que cualquier otro investigador pueda evaluar el trabajo.

Esta técnica sirve de apoyo cuando se realiza la codificación o categorización, donde surgen interrogantes, ideas y conceptos, que ilustran lo planteado en el estudio, por lo que resulta indispensable escribirlos para que no se olvide, ayudando a identificar unidades y categorías de significado, por lo que es importante registrar la fecha de la anotación, incluir la referencia o fuente, marcar las ideas en los encabezados, identificar el código, usar diagramas, esquemas y matrices si es necesario y registrar las reflexiones.

\subsection{Memo analítico}

El memo analítico documenta decisiones o definiciones hechas al momento de analizar los datos. Desde cómo surge una categoría, hasta el código que se le asigna o el establecimiento de una regla de codificación. Según Coleman y Unrau (2005), citado por Hernández y otros (2006:448) los memos analíticos se elaboran con fines de triangulación o auditoría entre investigadores, para que otras personas puedan ver lo que se hizo y cómo se hizo.

Al respecto, Grinnell y Unrau (2007) citado por el mismo Hernández sugiere el siguiente esquema: 1) memos, anotaciones o comentarios acerca del método de análisis, 2) memos sobre los problemas durante el proceso, 3) memos en relación con la codificación, 4) memos respecto a ideas y comentarios de los investigadores (incluyendo diagramas, mapas conceptuales, dibujos, esquemas, matrices), 5) memos sobre el material de apoyo localizado (fotografías, videos, etcétera) y 6) memos relacionados con significados, descripciones y conclusiones preliminares.

\subsection{Inducción analítica}

La inducción analítica es un procedimiento de tratamiento de la información recopilada cuyas primeras referencias se remontan a Znaniecki (1934), quien resalta que la inducción analítica 
considera la importancia de la percepción de la experiencia analizada por los participantes. Afirmó que "todos los hechos sociales son creados por los actores sociales y solo pueden entenderse desde su propia perspectiva, la inducción analítica es un procedimiento para verificar teorías y proposiciones."

\subsection{Codificación cualitativa}

En la mayoría de los estudios cualitativos se codifican los datos para tener una descripción más completa e ir eliminando la información de menor relevancia. Para Hernández y otros (2006: 448) en la codificación cualitativa el investigador considera dos segmentos de contenido, los analiza y compara. Si son distintos en términos de significado y concepto, de cada uno induce una categoría, si son similares, induce una categoría común. Los códigos por su parte identifican a las categorías que emergen de la comparación constante de segmentos o unidades de análisis.

Asimismo, refiere que la codificación tiene dos planos o niveles: en el primero, se codifican las unidades en categorías; en el segundo se comparan las categorías entre sí para agruparlas en temas y buscar posibles vinculaciones. Esta implica, además de identificar experiencias o conceptos en segmentos de los datos (unidades), tomar decisiones acerca de qué piezas "embonan" entre sí para ser categorizadas, codificadas, clasificadas y agrupadas para conformar los patrones que serán empleados con el fin de interpretar la información.

De hecho, en esta técnica de comparación constante, las categorías son conceptos, experiencias, ideas, hechos relevantes y con significado y deben guardar una relación estrecha con la información.

\subsection{Descripción densa}

Para algunas investigaciones cualitativas, la descripción es más profunda. Considerando a Geertz (1987: 302) "escribir y analizar las estructuras significativas de la experiencia, tales como son aprehendidas por los miembros de una determinada sociedad en un determinado momento, en una palabra, una fenomenología científica."

Se puede decir entonces, que la manera de plasmar perspectivas es a través de la descripción densa, en la cual la tarea central del investigador es generar sentido a partir de una descripción a escala micro y macro de una situación local.

En ese sentido, se debe hacer un estudio detallado que parta de un conocimiento abundante sobre lo pequeño, para arribar a conclusiones más amplias o abstractas y que se apoye en la teoría de una manera limitada en tanto esta le sirva para comprender densamente los fenómenos que ha estudiado de forma etnográfica.

\subsection{Análisis de contenido y análisis del discurso}

La necesidad de interpretar y comprender textos y discursos como parte de los estudios interpretativos ha tenido diversas formas y manifestaciones a lo largo de la historia de la 
investigación cualitativa. Krippendorff (1980), la Iglesia de registrar las indagaciones empíricas sobre el contenido de las comunicaciones, indican como primer ejemplo del análisis de contenido.

Basándose en dicho autor, se puede decir que este tipo de análisis es una técnica de interpretación y comprensión de textos escritos, orales, filmados, fotográficos, transcripciones de entrevistas y observaciones, discursos, documentos, es decir, todo tipo de registro teniendo en cuenta el contexto en el que se produce.

\subsection{Perspectiva de mapeo}

Esta técnica es muy utilizada en la actualidad. Para Schettini (1998), esta perspectiva se centra en las relaciones que construimos a partir de lo dicho, de los discursos de los sujetos (tanto lo que dicen de los otros, como lo que creen que los otros dicen de ellos o lo que creen que los otros dicen de sí mismos como lo que dicen de sí mismos.

Siguiendo dichas ideas, se menciona a Paulo Freire (1993), quien al sociograma lo denominaba mapeo, que evidentemente es un análisis de redes y la construcción de un mapa social, el cual ayuda a profundizar en los vínculos, las relaciones entre los actores sociales, entre las organizaciones, ya sean institucionales, comunitarias; en fin, todo tipo de relación entre los actores tanto de los que participan en la investigación, como la de aquellos, que no participando se relacionan de alguna manera con alguno de los sujetos de la investigación.

\subsection{La triangulación}

La Triangulación es llamada también "convergencia metodológica", "método múltiple" y "validación convergente", pero en todas estas nociones subyace el supuesto de que los métodos cualitativos y cuantitativos deben ser considerados no como campos rivales, sino como complementarios, tal como lo hace saber, Vasilachis I. (1992:36), citado a Jick (1979), porque refiere que en todos los diversos diseños de triangulación está implícita la asunción básica de que su efectividad se basa en la premisa de que las debilidades de cada método individual van a ser compensadas por la fortaleza contra-balanceadora del otro.

Al respecto, Ruth \& Finol (2009) puntualizan que "la triangulación en la investigación social presenta muchas ventajas porque el utilizar diferentes métodos, éstos actúan como filtros a través de los cuales se capta la realidad de modo selectivo".

Efectivamente, esta técnica de análisis se centra en el contrastar visiones o enfoques como una herramienta enriquecedora que le confiere a un estudio rigor, profundidad, complejidad y permite dar grados variables de consistencia a los hallazgos y a la vez permite reducir sesgos y aumentar la comprensión del fenómeno estudiado.

De acuerdo a la misma Vasilachis I. (1992:37), existen cuatro tipos básicos de triangulación: de datos (de tiempo, espacio y de personas), de investigadores, teórica y metodológica (intrametodologica y intermetodologica); sin embargo, son de igual nivel, lo que determina que algunas clases estén determinadas dentro de las otras, o que la elección de algunos de estos

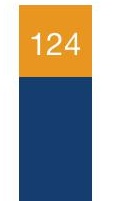


es el criterio derivado de la reflexión epistemológica que tendría el investigador, basados en: la triangulación teórico-metodológica y la triangulación de datos y de investigadores.

\section{Conclusiones}

Cualquiera sea la técnica e instrumento de recolección de información que se use en una investigación cualitativa, siempre conduce a un proceso para analizar desde una interpretación lo indagado por el investigador. Al analizar y procesar, se hace una selección particular de la información y se apoya no solo en la experiencia de vida, nuestro mundo sociocultural, sino también en la intuición y, fundamentalmente, en los propósitos de premisas del estudio.

Se debe, por ello, al analizar, agudizar los sentidos, ya que, la mayoría de las veces, se estará haciendo desde nuestro marco sociocultural que, por lo general, no es el de aquellos con quienes se realiza la investigación. Es importante ver la posición social que ocupan las personas, tanto de los investigadores, como de los sujetos o participantes con los que se trabaje, teniendo en cuenta que distintas palabras construyen distintas realidades.

Partiendo del desarrollo del presente artículo, se puede considerar como parte de la formación auto-reflexiva y critica del científico para la selección de la técnica o el instrumento de recolección de información en una investigación cualitativa, siendo otra génesis en el proceso en la obtención del conocimiento, donde él y los sujetos se retan y cuestionan el por qué y para qué de lo que sabe y se puede saber, y las razones por las cuales quiere apropiarse individual y socialmente de información. 


\section{Referencias}

Arias, F. (2006) El Proyecto de Investigación: Introducción a la Metodológica Científica (5ta edición) caracas: Espíteme.

Campoy, T \& Gomes, E. (2009). Técnicas e instrumentos cualitativos de recogida de datos. Manual básico para la realización de tesinas, tesis y trabajos de investigación.

Freire, P. (1993). Pedagogía de la esperanza: un reencuentro con la Pedagogía del Oprimido. Siglo XXI Editores, S.A., México.

Geertz. (1988). La interpretación de las culturas, Editorial Gedisa. España.

González, M. (1987). El taller de los talleres. Siglo XXI Editores, S.A., México.

Hernández, R., Fernández, C., \& Baptista, P. (2010). Metodología de la

Investigación. Quinta Edición. DERECHOS RESERVADOS @ 2010, 2006, 2003, 1998, 1991 respecto a la quinta edición por: McGRAW-HILL / INTERAMERICANA EDITORES, S.A. DE C.V.

Hernández, M. (2009). Exclusión Social en la Región de Murcia, Editum: Murcia

Ibáñez, J. (1992). La guerra incruenta entre cuantitativistas y cualitativistas. Editorial: Román, España.

Krippendorff K. (1980) "Content análisis: an introduction to its methodology", Sage Beverly Hills, California.

Martínez, M. (1999) La investigación cualitativa etnográfica en educación. Editorial: Trillas. México.

Morocho Espinoza, I. M. (2011). Elaboración y Aplicación de Instrumentos de Evaluación a los Indicadores Esenciales de Evaluación según la Reforma Curricular del 2010, en el área de Ciencias Naturales, para los niños de cuarto año de básica de la Escuela Manuel Utreras Gómez. Cuenca: Universidad Politécnica Salesiana.

Quintana, A. (2006). Un Modelo de aproximación empírica a la investigación en psicología y ciencias humanas. Revista Peruana de Psicología. Perú.

Ramírez K. (2017). El registro anecdótico en la evaluación formativa oral del idioma inglés. Universidad Central del Ecuador. Facultad de filosofía, letras y ciencias de la educación carrera inglés. Quito 2017.

Santana, L. (2010). Investigación Cualitativa. La vía más idónea para interpretar y comprender la dinámica social. Venezuela. Valencia. Estado Carabobo. 
Santos G (1993). Hacer visible lo cotidiano. Teoría y práctica de la evaluación cualitativa de centros escolares. Editorial Akal. Madrid.

Schettini, P (1998). Notas sobre los desafíos a la hora de analizar datos cualitativos o de cómo los investigadores construimos representaciones. En II Jornadas sobre Etnografía y Métodos Cualitativos.

Vargas X. (2010). ¿Cómo hacer investigación cualitativa? Una guía práctica para saber que es la investigación en general y como hacerla. (con énfasis en las etapas de la investigación cualitativa) (apropiada para quién hace investigación por primera vez). Unidad académica de contexto academia para el estudio de la interpretación y significación del hábitat departamento del hábitat y desarrollo urbano ITESO.

Vacilachis, I. (1992). Métodos Cualitativos I. Los problemas teórico- epistemológicos. Buenos Aires. Centro editor de América Latina.

Vallejo, R., \& de Franco, M. F. (2009). La triangulación como procedimiento de análisis para investigaciones educativas. Redhecs, 7(4), 117-133.

Znaniecki, F. (1934). The method of sociology. New York: Farrar \& Rinehar. 
Copyright (c) 2021 Maream José Sánchez Bracho, Mariela Fernández y Juan Díaz

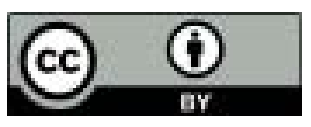

Este texto está protegido bajo una licencia internacional Creative Commons 4.0.

Usted es libre para Compartir-copiar y redistribuir el material en cualquier medio o formato - y Adaptar el documento - remezclar, transformar y crear a partir del material-para cualquier propósito, incluso para fines comerciales, siempre que cumpla las condiciones de Atribución. Usted debe dar crédito a la obra original de manera adecuada, proporcionar un enlace a la licencia, e indicar si se han realizado cambios. Puede hacerlo en cualquier forma razonable, pero no de forma tal que sugiera que tiene el apoyo del licenciante o lo recibe por el uso que hace de la obra.

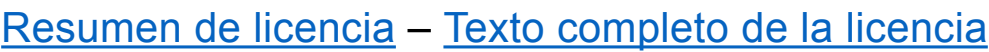

